\title{
FOREWORD
}

\section{Special Section on Networking Technologies for Overlay Networks}

\begin{abstract}
An overlay network is a logical high-layer network built over the well-established IP network for creating new networkbased services. Owing to its capabilities of a flexible use of the underlying networks and fast deployment of new services, various overlay networking technologies like P2P (peer-to-peer network) and IP-VPN (virtual private network) have been actively developed and introduced into the real world. However, at the same time, it is now recognized that it has a large influence on the design and management methodologies of the underlying IP networks.
\end{abstract}

The purpose of this special section aims to present the latest trend of overlay networking technologies and to promote further research and development in the field. Of course, a concept of overlay networking is not new in the layered network architecture, and this section is devoted to the overlay network over IP. Accordingly, we excluded those papers treating traditional aspects of the overlay networks. Nevertheless, we had received 45 submissions. After careful consideration and intensive review and discussion by the editorial committee, 17 papers have been selected for publication. Those papers cover central issues of new overlay networking technologies; resource finding/sharing/management, routing protocols, traffic engineering, new services, and security. Two invited papers are also solicited to provide future perspectives on overlay networks. The editorial committee hopes that these sophisticated papers will contribute to the further progress of overlay networks and future rich network services.

Last, all members of the editorial committee, who volunteered from the Technical Committee on Information Networking and the Technical Committee on Communication Quality of the IEICE Communication Society, would like to express our sincere appreciation to all of the authors and reviewers for their contributions to make this special section a successful one.

Special Section Editorial Committee Members

Guest Editors Hideki Nojiri (NTT), Masato Matsuo (NTT)

Guest Associate Editors Kenji Ishida (Hiroshima City Univ.), Hiroyuki Ibe (Toshiba Corp.), Hiroyuki Ohsaki (Osaka Univ.), Takashi Okuda (Aichi Prefectural Univ.), Yoshikuni Onozato (Gunma Univ.), Kenji Kawahara (Kyushu Inst. Tech.), Takumi Kimura (NTT Com.), Go Hasegawa (Osaka Univ.), Toru Hasegawa (KDDI Lab.), Hiroki Horiuchi (KDDI Lab.), Takumi Miyoshi (Shibaura Inst. Tech.), Tutomu Murase (NEC Corp.)

Masayuki Murata and Takeo Abe, Guest Editors-in-Chief

\begin{abstract}
Masayuki Murata (Fellow)
received the M.E. and D.E. degrees in Information and Computer Sciences from Osaka University, Japan, in 1984 and 1988, respectively. In April 1984, he joined Tokyo Research Laboratory, IBM Japan, as a Researcher. In September 1987, he moved to Osaka University. In April 1999, he became a Professor of Osaka University, and currently he is with Graduate School of Information Science and Technology, Osaka University. He has more than four hundred papers of international and domestic journals and conferences. His research interests include computer network architecture and related topics including photonic networks, sensor networks, and mobile ad hoc networks. He is a member of IEEE, ACM, The Internet Society.
\end{abstract}

Takeo Abe (Member)

received the B.E., M.E., and Dr. Eng. degrees in Applied Mathematics and Physics from Kyoto University, Kyoto, Japan, in 1978, 1980, and 1998, respectively. In 1980, he joined the Musashino Electrical Communication Laboratory of NTT Public Corporation (now NTT), where he engaged in research on network reliability, traffic management, and congestion control. Currently, he is a professor of healthcare informatics at Tokyo Healthcare University. Dr. Abe received the IEICE Young Engineer Award in 1988 and has been chair of IEICE Technical Committee on Communication Quality since 2005. He is a member of IEEE, HIMSS, ORSJ.
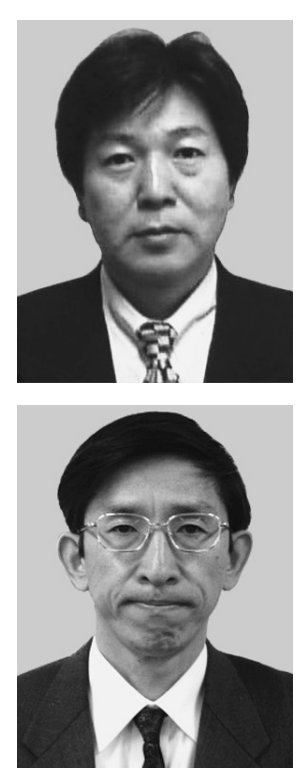